\title{
A Master Curve for the Size and Strain Rate Dependent Large \\ Deformation Behavior of PS Nanofibers at Room Temperature
}

\author{
Pavan V. Kolluru and Ioannis Chasiotis* \\ Aerospace Engineering, University of Illinois at Urbana-Champaign, Urbana, IL 61801, USA.
}

\begin{abstract}
Unlike the nominally brittle behavior of bulk polystyrene (PS), PS fibers with particular combinations of molecular weight (MW) and submicron scale diameters exhibit stable necking followed by pronounced strain-hardening, resulting in simultaneous increase in strength and ductility. The ratio, Dnorm, of the fiber diameter, D, to the intrinsic macromolecular length scale described by the root-mean-square end-to-end chain distance, $\mathrm{Ree}_{\mathrm{ee}} \mathrm{MW}$, has been shown to be an effective scaling parameter to determine the initiation and evolution of necking and strain hardening in submicron scale unoriented PS fibers. In this study, the room temperature large deformation response of PS nanofibers is quantified for the first time over a broad range of strain rates between $10^{-4}-10^{2} \mathrm{~s}^{-1}$. It is shown that for all combinations of MW in the range 123,000$2,000,0000 \mathrm{~g} / \mathrm{mol}$ and $\mathrm{D}$ in the range $200-750 \mathrm{~nm}$ that satisfy $\mathrm{D}_{\text {norm }}<10$, an increasing strain rate results in a monotonic increase of the stress amplitude without any reduction in the fiber stretch ratio to failure. Furthermore, the experimental stress vs. stretch ratio curves for $\mathrm{D}_{\text {norm }}<10$ obeyed a multiplicative decomposition of stress into a shape and a rate component. This decomposition permits the construction of a normalized stress vs. strain master curve that captures well both the size effects, originating in the relative molecular and specimen length scales, and the strain rate effects on the mechanical behavior of PS fibers at room temperature. KEYWORDS: Necking; Strain hardening; Electrospinning
\end{abstract}




\section{Introduction}

While amorphous polymers are intrinsically tough under compressive loading, they display one of two distinct types of deformation when subjected to tension at the glassy state: (i) immediate failure after yielding due to severe localization of plastic deformation, e.g. polystyrene (PS) or polymethyl methacrylate (PMMA), or (ii) sustained large deformation after stabilization of strain localization, e.g. polycarbonate (PC) [1]. Harnessing the intrinsic ductility of the former class of polymers under tensile loading has been a topic of extensive research. To this goal, the composition of glassy polymers has been modified, e.g., by the addition of a rubbery second phase such as polybutadiene [2-5] or cavitating rubber particles, by the reduction of intrinsic softening via mechanical rejuvenation [6], or via orientation of polymer molecules by melt stretching [7]. Most of these methods have produced a modest increase in ductility $(<50 \%)$ but often compromised the strength and stiffness of the neat polymer.

Recent room temperature (RT) uniaxial tension experiments with individual PS nanofibers have shown that the synergistic coupling between the intrinsic molecular length scale, as described by the root-mean-square end-to-end distance of an unperturbed polymer chain $\left(R_{\mathrm{ee}}\right)$, and the specimen structural length scale, as described by the fiber diameter $(D)$, could be exploited to enable a brittle-to-ductile transition in PS nanofibers [8], when the ratio of $D$ to $R_{\mathrm{ee}}$ ( $D_{\text {norm}}$ ) falls below $\sim 18$. This transition results in simultaneously increased tensile strength (by as much as $\sim 350 \%$ ) and ductility (by as much as $4,000 \%$ compared to the bulk brittle behavior) compared to bulk-scale PS specimens subjected to tension. This radical modification of the tensile behavior of submicron PS fibers was controlled by adjusting $D_{\text {norm }}$ without altering the polymer composition or structure. $D_{\text {norm }}$ expresses the fiber diameter as a multiple of the molecular length scale $\left(R_{\mathrm{ee}}\right)$ : a decreasing value of $D_{\text {norm }}$ represents the influence of the 
increasing fraction of free-surface chain segments which have reduced molecular constraints, on the effective mechanical behavior of a fiber.

This study focuses on the unexplored and intriguing viscous nature of the large plastic

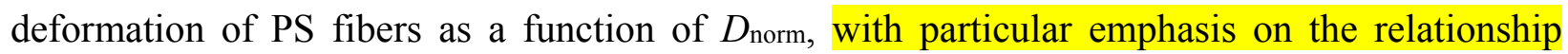
between the applied strain rate and the range of $D_{\text {norm, }}$ which results in complete necking and subsequent strain hardening. Amorphous polymers exhibit time and temperature dependent mechanical behavior due to the contribution of different relaxation mechanisms taking place at various time and length scales, ranging from fast segmental scale motions such as side-group rotation, to relatively slower and larger scale cooperative molecular motions $[9,10]$. For instance, yielding of glassy polymers can be understood as the result of the competition between material softening and hardening, each having a different strain rate dependence according to the contribution of $\alpha$ - and $\beta$-relaxations [11,12]. Although numerous experimental and modeling studies have focused on elucidating the viscous character of elasto-plastic deformation processes and failure mechanisms in bulk amorphous polymers [11-17], very few studies have explored the rate dependent mechanical behavior of submicron scale specimens [18-20] despite overwhelming evidence indicating that amorphous polymeric nanostructures have different relaxation spectra compared to bulk, such as broadening and/or shift of the $\alpha$ - regime [21-28]. Experiments on the thermo-viscoelastic behavior of freestanding ultrathin films of amorphous PS and poly(vinyl acetate) (PVAc) $[18,29]$ alluded that, contrary to expectations about an increased mobility of polymer chain segments at the film free surface, many relaxation modes (especially the slower relaxation modes) are likely suppressed at the film free surface. Similarly, it has been shown that the slowest characteristic relaxation times of as-spun polyacrylonitrile (PAN) nanofibers display 
further retardation with decreasing fiber diameter, which originates in the increased fraction of surface chain segments in smaller diameter fibers [30].

This study focuses on elucidating the viscoplastic response of individual PS nanofibers at room temperature as a function of molecular and specimen dimensions and the rate of loading. Specifically, the effects of strain rate on the stability of post-yield necking, the stretch ratio due to necking, and the subsequent strain hardening response are studied as a function of $D_{\text {norm. }}$. For the range of strain rates explored in this study, the strain rate sensitivity of yielding and post-

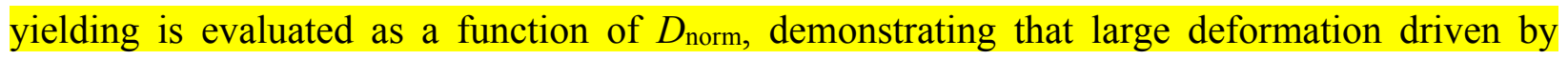
stable necking occurs for $D_{\text {norm }}<10$. Finally, a master curve that simultaneously captures the molecular length scale and specimen size, and the effect of strain rate on the tensile stress vs. strain response of the PS nanofibers studied in this work is presented.

\section{Materials and Methods}

\subsection{Synthesis of Polystyrene (PS) Nanofibers}

The extent of spatial confinement of macromolecules in submicron scale fibers is governed by the competition between two length scales: the fiber diameter, $D$, and the molecular length scale which is related to molecular weight, $M W$. To this effect, the molecular length scale can be described by $R_{\text {ee }}$ which is a function of $M W$ [8]. In order to study the relative role of these length scales, PS fibers were synthesized by electrospinning solutions of monodisperse linear atactic PS powders (Pressure Chemicals Inc.) in $N$-, $N$-dimethylformamide (DMF) according to the conditions described in Table 1. All fibers were electrospun at $1 \mathrm{kV} / \mathrm{cm}$ average electric field ( $25 \mathrm{kV}$ bias between the source and the collector that were separated by $25 \mathrm{~cm}$ ) in room temperature conditions $\left(22 \pm 3{ }^{\circ} \mathrm{C}\right.$ and $19 \pm 2 \%$ relative humidity). The as-spun PS fibers were 
annealed in their freestanding configuration at $10-25{ }^{\circ} \mathrm{C}$ above their bulk $T_{\mathrm{g}}$, and for durations that were longer than the reptation times of bulk-scale specimens, to devoid them of molecular orientation and the undesirable axially corrugated surface morphology arising from electrospinning [8]. Prior work by this group has shown that the geometry of the fiber crosssection and the fiber surface morphology define the evolution of the mechanical deformation of polymeric nanofibers [31]. The annealing times for different molecular weights are presented in Table 1 as multiples of the reptation times of the corresponding bulk scale samples $\left(\tau_{\mathrm{d}-b u l k}\right)$. Naturally, these times are overestimates of the annealing times required for freestanding nanofibers which allow for fast relaxations compared to bulk PS. While the diameters of all PS fibers tested in this work were in the range of $150-900 \mathrm{~nm}$, the electrospinning community typically refers to them as nanofibers, which term is also used henceforth in this paper.

Table 1. Electrospinning and annealing parameters used in the preparation of PS nanofibers. The polydispersity index (PDI) indicates the extent of dispersion in macromolecular chain lengths; a value of unity indicates no dispersion of the macromolecular length scale.

\begin{tabular}{|c|c|c|c|c|c|}
\hline \multirow[b]{2}{*}{$\begin{array}{c}\text { Molecular Weight } \\
\text { (g/mol) }\end{array}$} & \multirow[b]{2}{*}{ PDI } & \multirow{2}{*}{$\begin{array}{c}\text { Solution } \\
\text { Concentration } \\
\text { (weight \%) }\end{array}$} & \multicolumn{2}{|c|}{ Annealing Conditions } & \multirow[b]{2}{*}{$\begin{array}{c}\text { Nanofiber } \\
\text { Diameter } \\
(\mathbf{n m})\end{array}$} \\
\hline & & & $\begin{array}{c}\text { Temperature } \\
\left({ }^{\circ} \mathrm{C}\right)\end{array}$ & $\begin{array}{c}\text { Time } \\
\left(\times \tau_{\text {d-bulk }}\right)\end{array}$ & \\
\hline \multirow[t]{2}{*}{123,000} & 1.06 & $30^{*}$ & 110 & 6.3 & $200-500$ \\
\hline & & 30 & 110 & 6.3 & $700-900$ \\
\hline \multirow[t]{2}{*}{400,000} & 1.06 & 15 & 120 & 8.3 & $150-250$ \\
\hline & & 20 & 120 & 8.3 & $300-600$ \\
\hline \multirow[t]{2}{*}{$2,000,000$} & 1.30 & 2.0 & 125 & 0.2 & $150-250$ \\
\hline & & 4.5 & 125 & 0.2 & $300-800$ \\
\hline
\end{tabular}

* Solution contained $1 \mathrm{wt} \%$ tetrabutyl ammonium chloride (TBAC) to reduce the fiber diameter. 


\subsection{Mechanical Experiments with Individual PS Nanofibers at Different Strain Rates}

The tensile behavior of individual annealed PS nanofibers was experimentally determined following a microelectromechanical systems (MEMS)-based mechanical testing methodology developed by this group before, which provides high resolution measurements of the applied force (engineering stress) and elongation (fiber stretch ratio) [32,33] at ambient conditions. The specific surface micromachined MEMS devices are comprised of the three components shown in Figure 1. A nanofiber is mounted across components (1) and (2), with component (1) fixed to the substrate carrier of the MEMS device and component (2) being freestanding. The freestanding components (2) and (3) are connected to each other through a set of parallel beams (only partially visible in Figure 1) that act as an integrated force sensor whose stiffness is calibrated as described in [34]. In an experiment, the MEMS substrate carrier and therefore, the device components numbered (1), are translated to the left by a PZT actuator in nanometer-level displacement increments, while component (3) is held stationary with the aid of an external probe (not visible in Figure 1). In the present study, actuation speeds in the range $15 \mathrm{~nm} / \mathrm{s}-6$ $\mathrm{mm} / \mathrm{s}$ were imposed with an external PZT actuator. These microscale tension experiments were performed under an optical microscope in ambient conditions $\left(23^{\circ} \mathrm{C}\right.$ and $20-30 \%$ relative humidity). Optical microscopy facilitated fast image acquisition rates of $\sim 40,000$ fps, thus enabling engineering strain rates as high as $10^{2} \mathrm{~s}^{-1}$ (the local strain rate during fiber necking is much higher.) The motion of each of the three components of the device shown in Figure 1 was calculated with $20 \mathrm{~nm}$ displacement resolution via Digital Image Correlation (DIC), which translates into $\sim 0.1 \%$ strain resolution and $<1 \mathrm{MPa}$ stress resolution for the particular nanofiber gauge length and loadcell stiffness. Figure 1 shows an example of the calculated displacements 
of different parts of the MEMS device, which are superposed onto an image of the MEMS device obtained during fiber testing.

Individual long PS nanofibers were isolated and cut into multiple segments of $100-150$ $\mu \mathrm{m}$ each. A section of 30-35 $\mu \mathrm{m}$ of each nanofiber segment served as the gauge section that was mounted onto the MEMS device using an epoxy adhesive, while the fiber segment outside the gauge section served the purpose of post-testing measurement of the initial fiber diameter. After testing, a $5 \mathrm{~nm} \mathrm{Au-Pd} \mathrm{layer} \mathrm{was} \mathrm{sputtered} \mathrm{onto} \mathrm{the} \mathrm{nanofibers} \mathrm{to} \mathrm{facilitate} \mathrm{SEM} \mathrm{imaging.} \mathrm{The}$ exact length of the nanofiber gauge section depended on looseness of the fiber during mounting on the MEMS device; the exact fiber gauge length was then determined at the point that the fiber began carrying load.

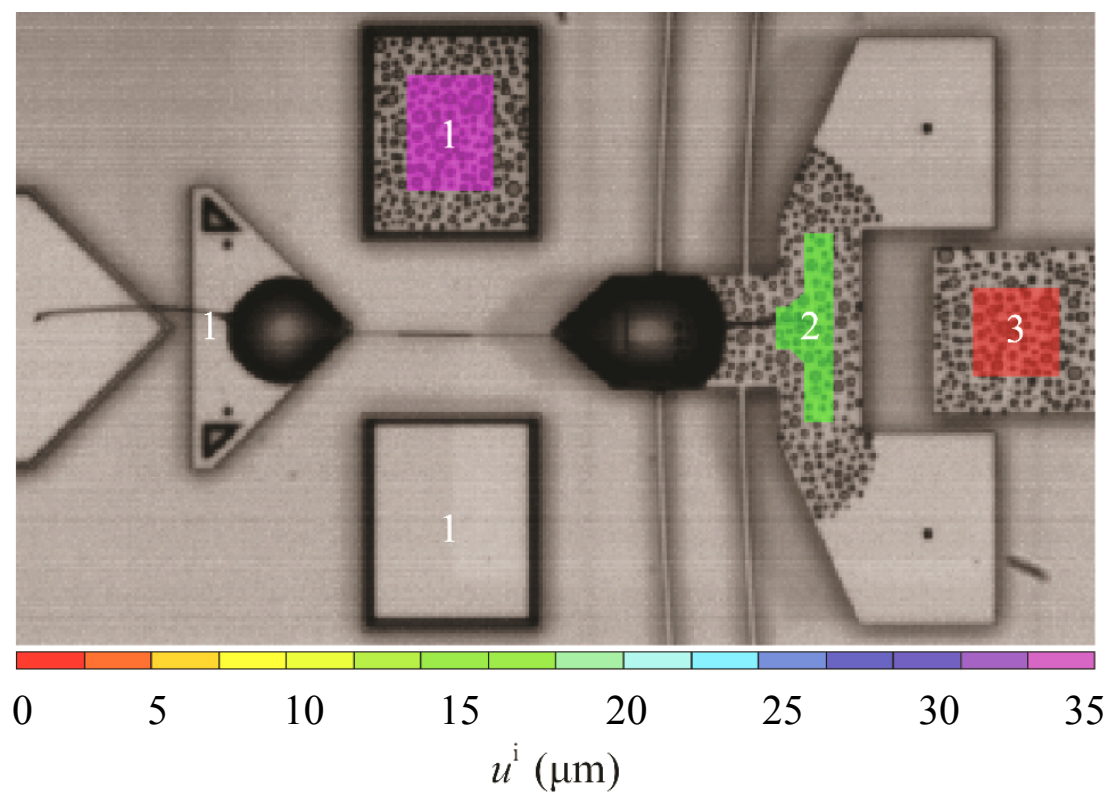

Figure 1. Optical micrograph showing the motion $\left(u^{1}, u^{2}, u^{3}\right)$ of components (1), (2) and (3) of a MEMS device during nanofiber testing. All the components labeled (1) are fixed to the underlying substrate and move together with the substrate. The displacements, $u^{\mathrm{i}}$, resolved with sub-pixel accuracy $(20 \mathrm{~nm})$ via DIC are used to compute the nanofiber elongation and the applied force. See also references [32,33]. An artificial speckle pattern was created on the surface of device components (1), (2), (3) using a focused ion beam (FIB). 


\section{Results and Discussion}

\subsection{Viscoplastic Behavior of PS Nanofibers}

The experimental results of the mechanical behavior of individual PS nanofibers are presented in the form of the engineering stress vs. stretch ratio at different engineering strain rates. The latter were calculated via linear fitting of the engineering strain vs. time data, as illustrated in Figure 2. Due to the compliance of the MEMS loadcell that is in line with the nanofiber specimen and post-yield strain localization in nanofibers, the engineering strain rates in the elastic regime is different from that in the post-yielding plastic regime. The engineering strain rate varies very little in the necking and hardening regimes, as shown in Figure 2. Therefore, henceforth, we refer to the elastic (pre-yielding) strain rate, $\dot{\varepsilon}_{\text {elastic, }}$ and the plastic strain rate, $\dot{\varepsilon}_{\text {plastic. }}$ The latter is given by the slope of a single linear regression fit line across the entire post-yield regime that includes necking and strain hardening, as the slopes of these two regimes were quite similar.

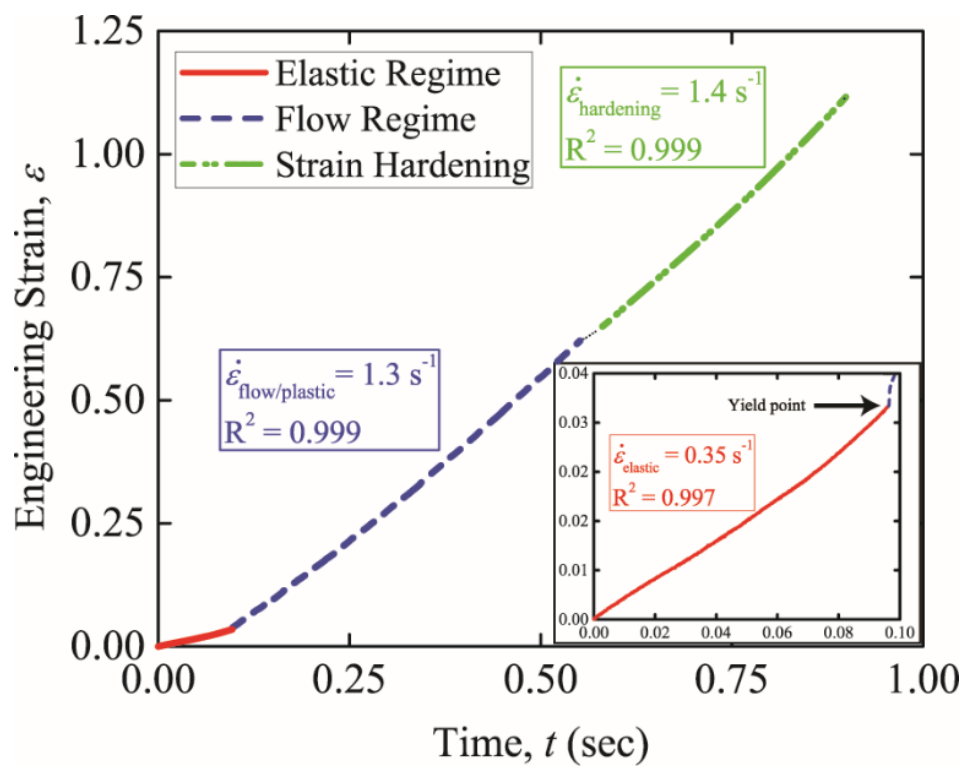

Figure 2. Example of calculation of strain rate from engineering strain vs. time data. The linear correlation coefficient $\left(\mathrm{R}^{2}\right)$ is also shown. The inset provides an enlarged view of the elastic regime. 
The ratio $D_{\text {norm }}=D / R_{\text {ee }}$, provides a measure of molecular confinement in individual nanofibers [8,35]. Smaller values of $D_{\text {norm }}$ imply larger fraction of polymer chain segments near or at the fiber free surface, which follow different molecular conformations compared to polymer chain segments in bulk [35-37], thus leading to increased spatial confinement that forces the polymer macromolecules to conform within the cross-section of a smooth fiber. Although $D_{\text {norm }}$ does not provide any details about the spatial distribution of molecular confinement, it helps to assess its importance in a set of nanofibers comprised of different molecular weights and diameters [8] and help to present the experimental results in a more systematic manner. Based on the experimental engineering stress vs. strain response of individual PS nanofibers the experimental results are divided into the two regimes of $D_{\text {norm }}<10$ and $D_{\text {norm }}>10$. All nanofibers with $D_{\text {norm }}<10$ demonstrated stable necking and pronounced subsequent strain hardening, while fibers with $D_{\text {norm }}>10$ either lacked the pronounced strain hardening regime after complete neck propagation, or at higher strain rates the fiber failed before the completion of neck propagation.

Representative engineering stress vs. stretch ratio curves obtained at different strain rates from PS nanofibers with $M W=123,000,400,000$ and 2,000,000 $\mathrm{g} / \mathrm{mol}$, all having $D_{\text {norm }}<10$, are shown in Figure 3. Notably, both the plastic stretch ratio accrued during necking $\left(\lambda_{\mathrm{h}}\right)$ and the overall stretch ratio at failure ( $\left.\lambda_{\text {failure }}\right)$ were quite independent of $\dot{\varepsilon}_{\text {plastic. }}$ The yield and flow stress values, however, increased with the applied strain rate, which is consistent with the notion that an increased rate of loading diminishes the time available for some relaxation processes. All PS nanofibers with $D_{\text {norm }}<10$ exhibited stable necking and pronounced post-neck hardening response thereafter, in the entire range of $\dot{\varepsilon}_{\text {plastic }}$ between $10^{-4}$ and $10^{2} \mathrm{~s}^{-1}$ in contrast to the room temperature tensile behavior of bulk PS at strain rates $10^{-4}-10^{-1} \mathrm{~s}^{-1}$ which fails at yielding [38]. 


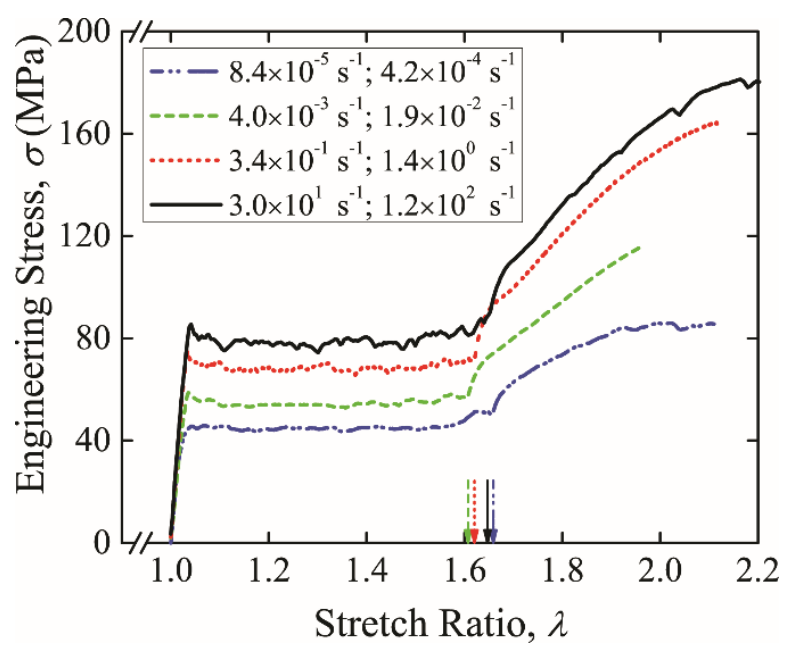

(a)

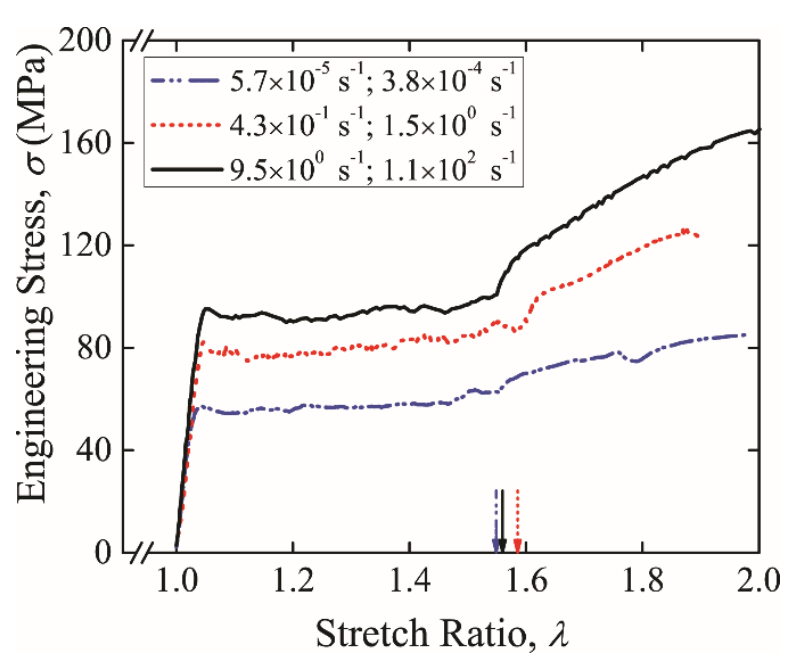

(c)

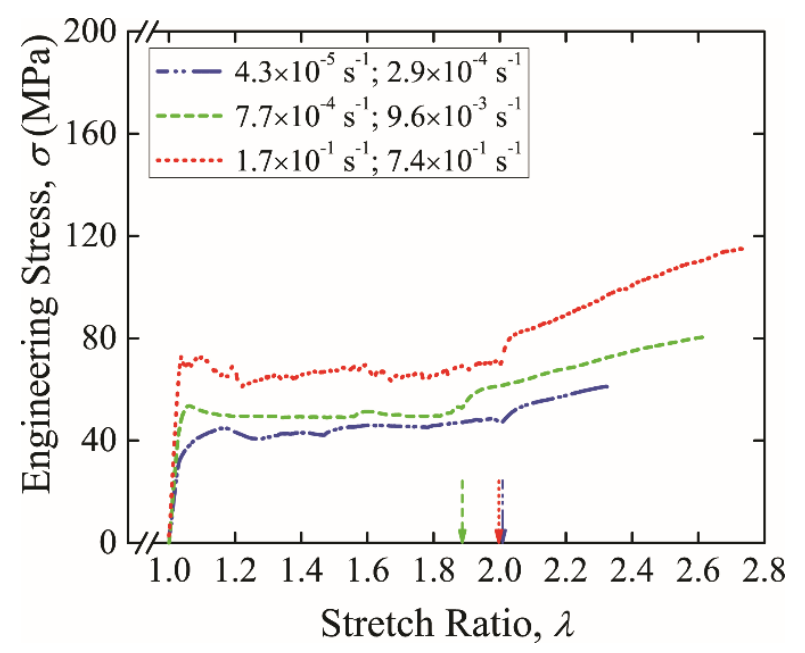

(b)

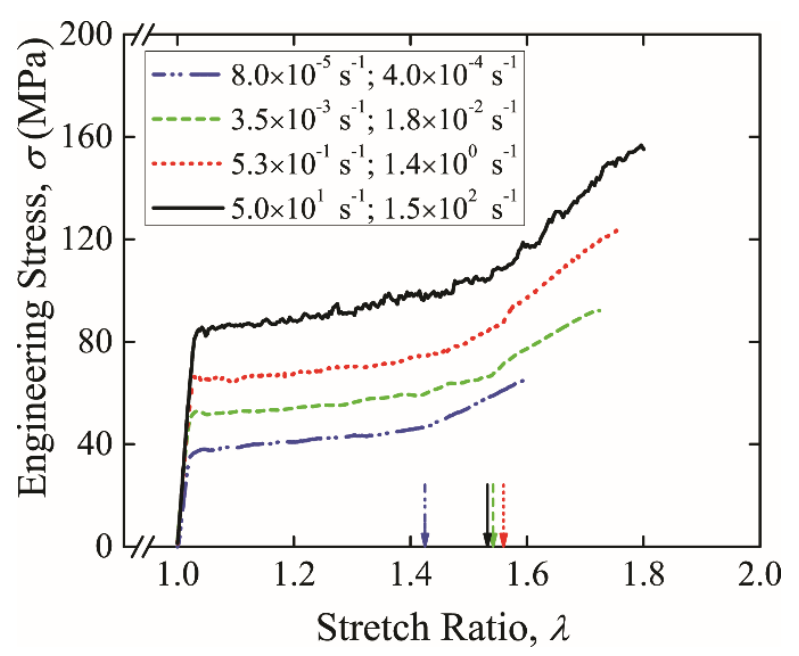

(d)

Figure 3. Engineering stress vs. stretch ratio curves at different engineering strain rates and $D_{\text {norm }}<10$. (a) $M W=2,000,000 \mathrm{~g} / \mathrm{mol}$ and $D=360 \mathrm{~nm}\left(D_{\text {norm }} \approx 3\right)$, (b) $M W=2,000,000 \mathrm{~g} / \mathrm{mol}$ and $D=750 \mathrm{~nm}$ ( $\left.D_{\text {norm }} \approx 6\right)$, (c) $M W=400,000 \mathrm{~g} / \mathrm{mol}$ and $D=350 \mathrm{~nm}\left(D_{\text {norm }} \approx 8\right)$, and (d) $M W=123,000 \mathrm{~g} / \mathrm{mol}$ and $D=$ $190 \mathrm{~nm}\left(D_{\text {norm }} \approx 8\right)$. The legends show $\left(\dot{\varepsilon}_{\text {elastic }} ; \dot{\varepsilon}_{\text {plastic }}\right)$. The arrows $(\downarrow)$ point to the value of $\lambda_{h}$. 


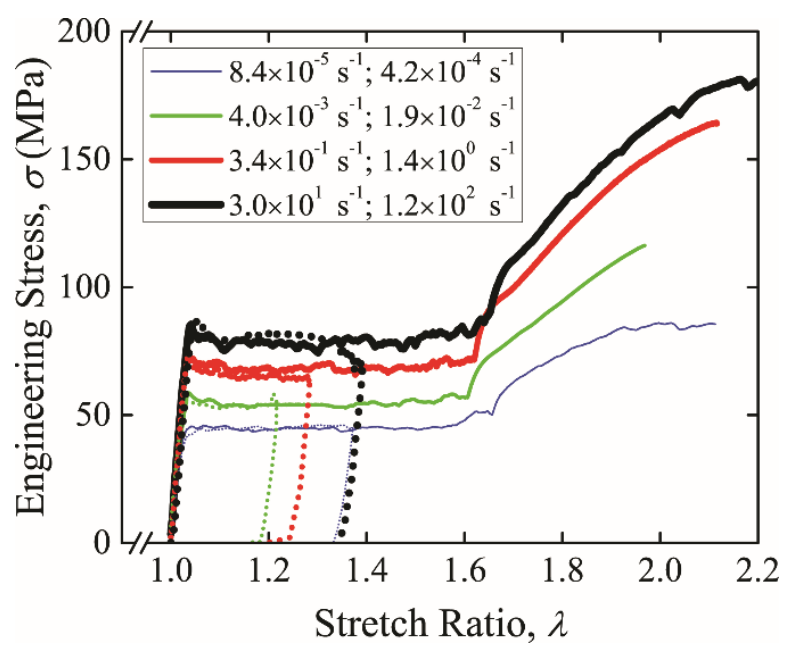

(a)

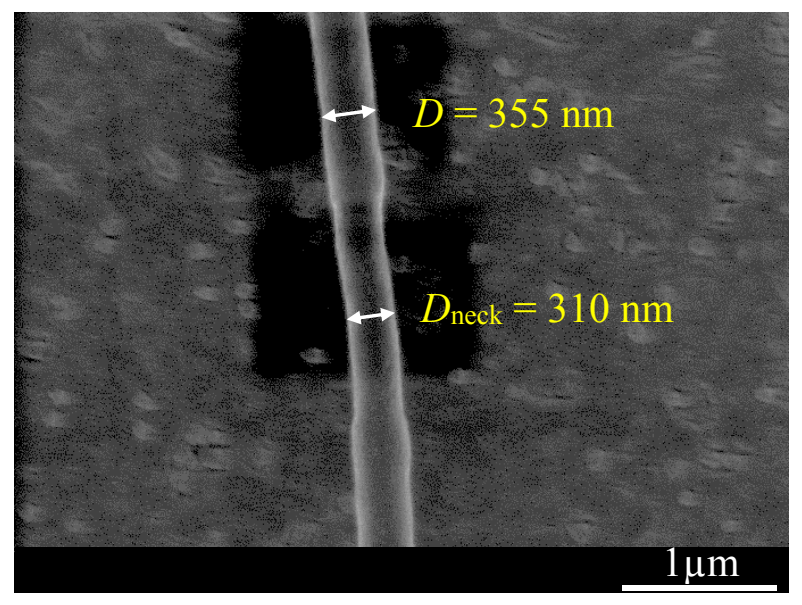

(b)

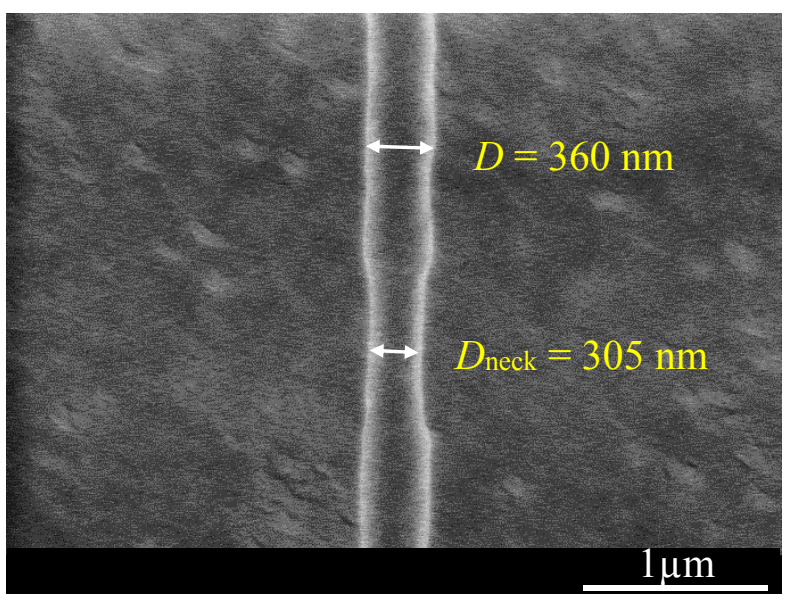

(c)

Figure 4. (a) Engineering stress vs. stretch ratio curves from eight segments of a PS nanofiber $(M W=$ 2,000,000 g/mol; $D=360 \mathrm{~nm} ; D_{\text {norm }}=3$ ), that were either stretched until failure (solid lines), or were tested in tension until the post-yield stress plateau, followed by unloading (dotted lines). Postmortem SEM images from interrupted tensile experiments, at the (b) slowest and (c) fastest strain rates in (a), showing that the ratio $D / D_{\text {neck }}$ is not affected by strain rate. The engineering stress vs. stretch ratio curves in (a) are indicative of the extremely consistent mechanical response of segments of the same nanofiber. Note that the accuracy in the measurement of the local fiber diameter and the variation of fiber diameter along its length is of the order of $5 \mathrm{~nm}$. 
Interrupted tension experiments with segments of the same PS nanofiber with $M W=$ $2,000,000 \mathrm{~g} / \mathrm{mol}$ and $D=360 \mathrm{~nm}$ were used to capture the formation and propagation of a stable neck in the stress plateau region, Figure 4(a). Postmortem SEM images, such as those in Figures 4(b,c), showed that the plastic neck diameter was independent of the applied strain rate. It is important to note that a rate-independent plastic neck diameter also implies that the stress vs. stretch ratio curves shown in Figures 3(a-d) would follow the same scaling with strain rate, if plotted with respect to engineering or true stress. The true stress in the necked region, in the plateau segment of the stress vs. strain curve, and in the subsequent hardening segment can be obtained approximately using interrupted experiments as described in [8].

In contrast to the consistent strain rate response of PS nanofibers with $D_{\text {norm }}<10$, the mechanical behavior of nanofibers with $D_{\text {norm }}>10$ demonstrated a variety of stress vs. stretch ratio curves with applied strain rate, except for the yield and flow stress that were consistent with the experiments on nanofibers with $D_{\text {norm }}<10$. For instance, Figure 5(a), a PS nanofiber with $D_{\text {norm }} \approx 11(M W=400,000 \mathrm{~g} / \mathrm{mol})$ demonstrated stable necking and mild post-neck hardening only at the two fastest strain rates, while failure occurred before complete neck propagation at the lowest strain rate of $\dot{\varepsilon}_{\text {plastic }}=5.8 \times 10^{-4} \mathrm{~s}^{-1}$, potentially due to the increased role of stress relaxation that reduced hardening. Similarly, the mechanical response of a PS nanofiber of $M W=$ $123,000 \mathrm{~g} / \mathrm{mol}$ and $D_{\text {norm }}=18$ shown in Figure 5(b) completely lacks post-neck hardening, and even demonstrates bulk behavior at the highest elastic strain rate of $29 \mathrm{~s}^{-1}$. Increasing the plastic strain rate by four orders of magnitude from $4.4 \times 10^{-4} \mathrm{~s}^{-1}$ to $1.4 \mathrm{~s}^{-1}$ also increased the fiber stretch ratio from $20 \%$ to $90 \%$, by allowing for stable neck propagation in longer segments of the fiber before failure. The limited propagation of a neck at the low strain rates indicates weak post-yield hardening which was not sufficient to maintain stable neck propagation along the entire fiber 
length. In this context, the fivefold increase in the post-yield plateau between $\dot{\varepsilon}_{\text {plastic }}=4.4 \times 10^{-4} \mathrm{~s}^{-1}$ and $\dot{\varepsilon}_{\text {plastic }}=1.4 \mathrm{~s}^{-1}$ implies that post-yield hardening increased with increasing strain rate, until a further increase in strain rate to $\dot{\varepsilon}_{\text {elastic }}=29 \mathrm{~s}^{-1}$ resulted in a ductile-to-brittle transition. Postmortem SEM image analysis showed that this early fiber failure was due to incipient localization of deformation, which could not stabilize into a neck, instead, leading to local fibrillation. A similar rate-dependent ductile behavior and a rate-induced brittle-to-ductile transition were previously reported for bulk PS coupons subjected to uniaxial tension at much higher temperatures of $65-85^{\circ} \mathrm{C}[38]$.

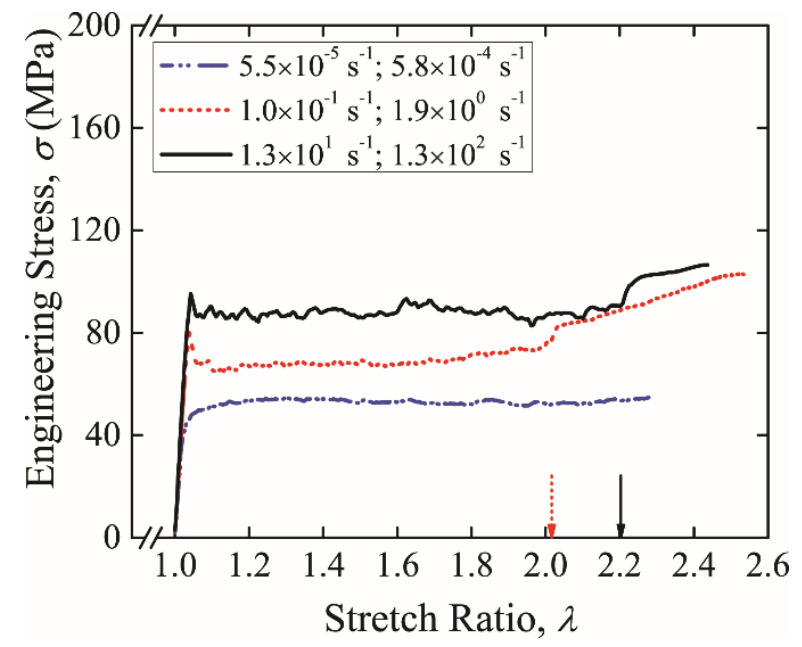

(a)

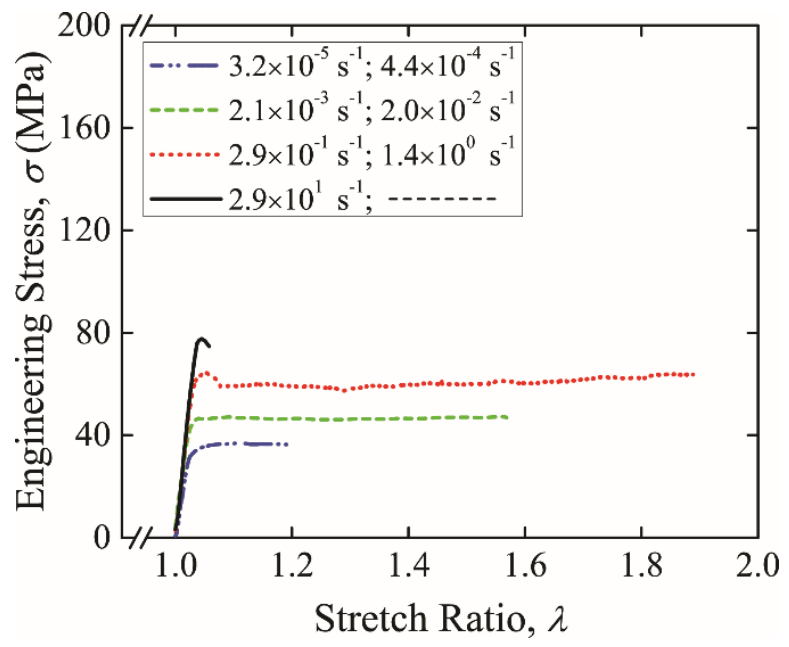

(b)

Figure 5. Engineering stress vs. stretch ratio curves of PS nanofibers with (a) $M W=400,000 \mathrm{~g} / \mathrm{mol}$ and $D$ $=475 \mathrm{~nm}\left(D_{\text {norm }} \approx 11\right)$, and (b) $M W=123,000 \mathrm{~g} /$ mole and $D=420 \mathrm{~nm}\left(D_{\text {norm }} \approx 18\right)$ for different strain rates (legends show $\dot{\varepsilon}_{\text {elastic }} ; \dot{\varepsilon}_{\text {plastic }}$ ). The arrows $(\downarrow)$ in (a) indicate $\lambda_{\mathrm{h}}$. Where arrows are missing, the nanofiber failed before the completion of neck propagation along its entire length. 


\subsection{Strain Rate Sensitivity of PS Nanofibers}

The strain rate sensitivity of stress, i.e. the increase in stress per unit increase in applied strain rate, is typically estimated for glassy amorphous polymers by Eyring's equation [39]:

$$
\sigma=\frac{\Delta H}{V^{*}}+\frac{k \cdot T}{V^{*}} \cdot \ln \left(\frac{\dot{\lambda}}{\dot{\lambda}_{o}}\right)
$$

where the activation energy, $\Delta H$, activation volume, $V^{*}$, and reference strain rate, $\dot{\lambda_{\mathrm{o}}}$, are fitting parameters. The slope of Equation 1 is a measure of the strain rate sensitivity. Under isothermal conditions, the slope of Equation 1 can also be used to calculate $V^{*}$. Figure 6 depicts the $D_{\text {norm }}$ dependence of $V^{*}$, calculated using the yield stress values (the stress at which neck initiation began) obtained from the plots in Figures 3 and 5. The fits show that all PS nanofibers had similar strain rate sensitivity at yielding irrespective of $D_{n o r m}$, and, as a result, the corresponding activation volume for yielding shown in Figure 6 was also quite independent of $D_{\text {norm }}$ in the range $3<D_{\text {norm }}<20$. The invariance of $V^{*}$ at the onset of yielding with $D_{\text {norm indicates that the }}$ molecular mechanism leading to the onset of yielding was the same for all nanofibers tested, namely independent of nanofiber diameter and $M W$ for the range of diameters and $M W \mathrm{~s}$ used in this study. Furthermore, this result confirms that the material state of all electrospun PS fibers after annealing was the same and did not depend on the specimen diameter or $M W$. 


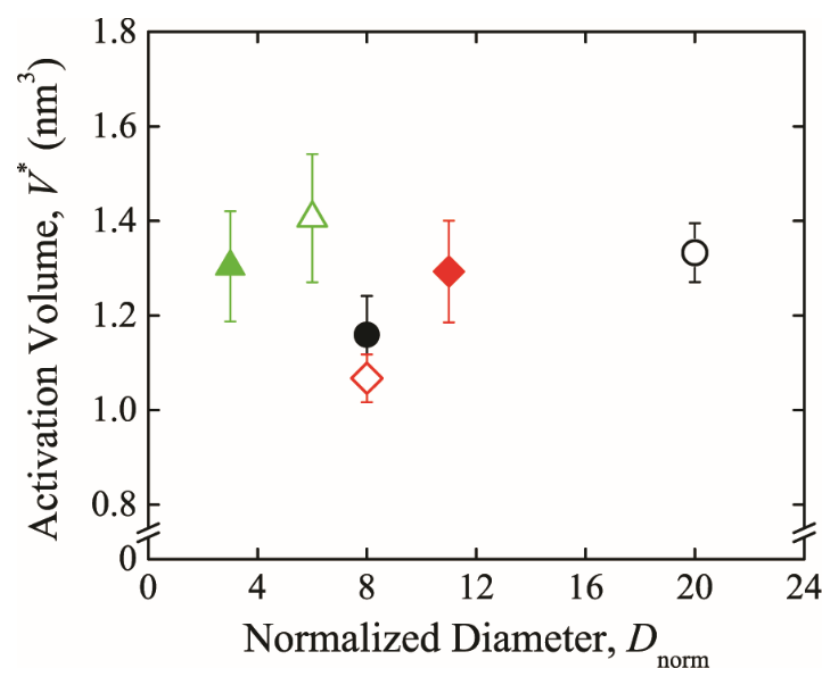

Figure 6. Activation volume $\left(V^{*}\right)$ vs. $D_{\text {norm }}$ obtained via Equation 1 using the yield stress values computed from the plots in Figures 3 and 5. Open and filled circles, triangles and diamonds represent data from nanofibers with $M W=123,000,400,000$ and 2,000,000 g/mol, respectively.

\subsection{Scaling of Strain Rate Dependent Post-yield Plastic Deformation}

While the strain rate sensitivity of yield stress was shown in the previous Section to be independent of $D_{\text {norm, }}$, the post-yield plastic deformation and strain hardening behavior of PS nanofibers did depend on $D_{\text {norm }}$. It was shown in Figure 3 that PS nanofibers with $D_{\text {norm }}<10$ exhibit stable necking and significant post-neck hardening, with strain rate insensitive stretch ratios marking the completion of necking and fiber failure, while the particular values of onset stress in each deformation regime varied with the applied strain rate. The invariance of fiber stretch ratio due to necking, coupled with the consistent increase of stress with applied strain rate provides a basis for scaling the stress vs. stretch ratio response with the corresponding yield or flow stress: For example, the engineering stress vs. stretch ratio curves for different strain rates in Figure 3(a) can be shown to collapse onto a single curve in Figure 7(a) where the engineering stress is normalized with the corresponding value of the flow/plateau stress. The deviation in the 
post necking hardening regime can be further reduced by normalizing with the yield stress (defined as the upper yield point), as shown in Figure 7(b). Similarly, the rest of the plots in Figure 3, all for $D_{\text {norm }}<10$, when normalized by the corresponding yield stress also collapse onto a single curve, as shown in Figure 8. Minor deviations in the post-neck strain hardening regime, particularly in Figure $8(\mathrm{c})$, arose from minor differences in $\lambda_{\mathrm{h}}$, as can be confirmed by the similarity of the hardening slopes in the normalized stress vs. strain response.

This scaling of the stress vs stretch ratio behavior is possible because the yield stress and the post-yield plastic deformation response of PS nanofibers have the same strain rate dependency. In fact, recent molecular simulations [40] and theoretical studies [41] have identified similar scaling for the stress vs. stretch ratio response of glassy polymers with respect to flow or yield stresses, thus signifying a multiplicative decomposition of stress into a shape and a rate component given by

$$
\sigma(\lambda, \dot{\lambda})=f(\lambda) \cdot \sigma_{\text {yield } / \text { flow }}(\dot{\lambda})
$$

where $f(\lambda)$ is the shape factor and the $\sigma_{\text {yield/flow }}(\dot{\lambda})$ is the rate component that describes the strain rate dependence of yield or flow stress. Similar scaling of stress with strain rate has been shown for bulk PS subjected to uniaxial compression [42], albeit in the narrow strain rate range of $10^{-4}-10^{-3} \mathrm{~s}^{-1}$ and for $\lambda<1.5$. However, in light of the slight, yet systematic, differences observed between scaled curves for $\lambda>1.5$, the authors in [42] argued that Equation 2 is not truly satisfied in the low strain rates used in their experiments. It was, thus, suggested that although scaling of strain hardening with flow stress may hold at $\dot{\varepsilon}>1,000 \mathrm{~s}^{-1}$ corresponding to molecular simulations [40,43], it might not apply at the low strain rates usually employed in compression experiments. By contrast, the experimental results presented in Figures 7 and 8 , show that Equation 2 does hold across a very wide range of elastic tensile strain rates between 
$10^{-4}$ and $10^{2} \mathrm{~s}^{-1}$, and in the neck transition region for rates in the approximate range of $10^{-2} \mathrm{~s}^{-1}$ $10^{4} \mathrm{~s}^{-1}$ as the neck transition length was about two orders of magnitude smaller than the initial fiber gauge length, thus the local strain rate during neck propagation in the neck transition region was $\sim 2$ orders of magnitude higher than the engineering strain rates calculated in Figure 2 [44].

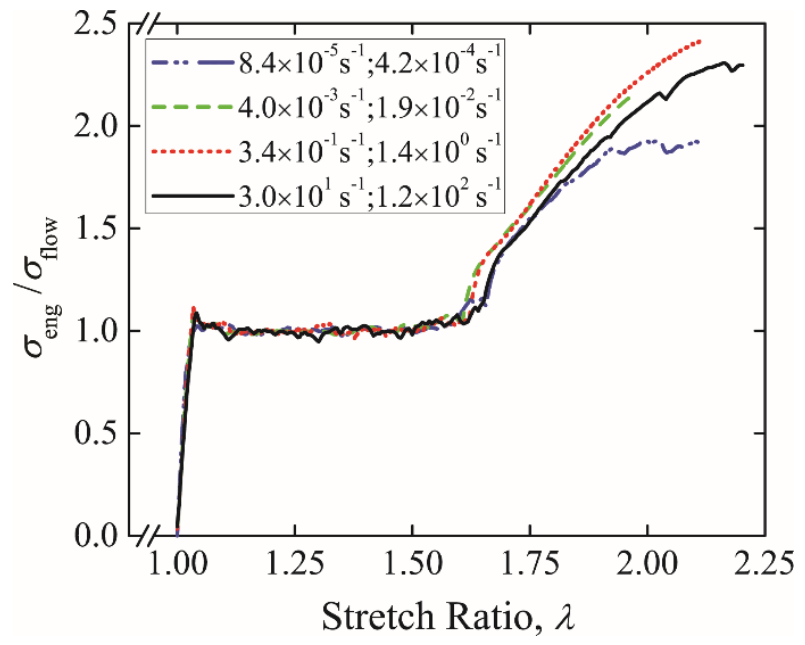

(a)

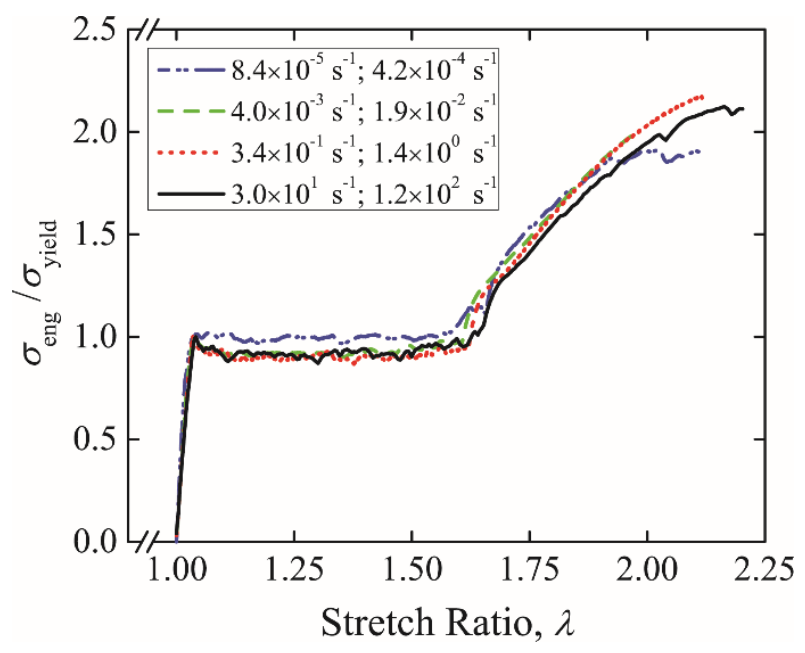

(b)

Figure 7. Engineering stress vs. stretch ratio curves in Figure 3(a) normalized by (a) flow/plateau stress and (b) yield stress. The legends show ( $\left.\dot{\varepsilon}_{\text {elastic }} ; \dot{\varepsilon}_{\text {plastic }}\right)$. 


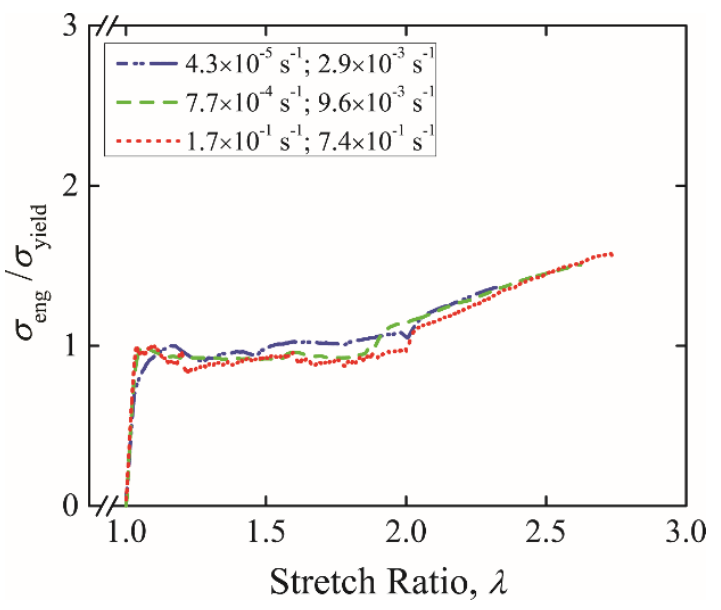

(a)

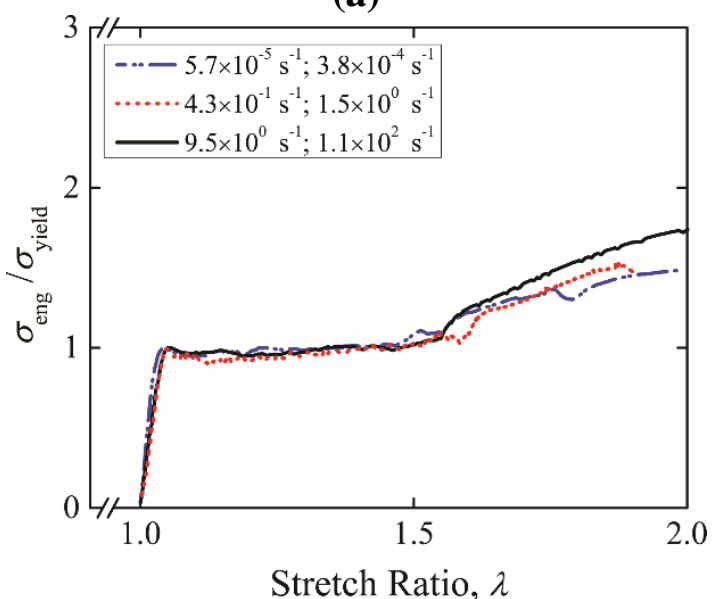

(b)

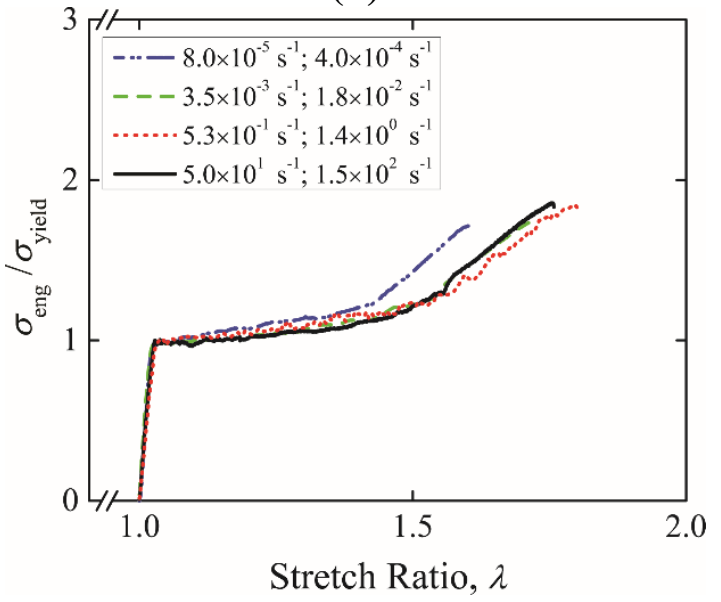

(c)

Figure 8. Engineering stress vs. stretch ratio, normalized with yield stress, for PS nanofibers with (a) $M W=2,000,000 \mathrm{~g} / \mathrm{mol}$ and $D=750 \mathrm{~nm}\left(D_{\text {norm }} \approx 6\right)$, (b) $M W=400,000 \mathrm{~g} / \mathrm{mol}$ and $D=$ $350 \mathrm{~nm}\left(D_{\text {norm }} \approx 8\right)$ and (c) $M W=123,000 \mathrm{~g} / \mathrm{mol}$ and $D=190 \mathrm{~nm}\left(D_{\text {norm }} \approx 8\right)$, based on the engineering stress vs. stretch ratio curves in Figures 3(b)-(d). The legends show ( $\dot{\varepsilon}_{\text {elastic }}$ ; $\dot{\varepsilon}_{\text {plastic }) \text {. }}$ 
The post-neck hardening regime in the $f(\lambda)$ curves in Figures 7(b) and $8(\mathrm{a}-\mathrm{c})$ does not exhibit any systematic trend with the applied strain rate, as also reported before in [42] for compression of bulk specimens. The current experimental data on the rate dependent tensile deformation of PS nanofibers show that (a) Equation 2 is applicable up to $\lambda \approx 2-2.5$, namely over a much wider range of stretch ratios than previously reported [40,42], and (b) the scaling law in Equation 2 is satisfied in the case of necking too, hence Equation 2 can be applied for normal or shear stress, as also proposed in [41]. This last argument is also supported by the fact that the neck characteristics (neck diameter and transition) for the same fiber were independent of strain rate. It is also important to emphasize that, although Equation (2) is implemented in Figures $7(\mathrm{~b})$ and $8(\mathrm{a}-\mathrm{c})$ using the engineering stress vs. stretch ratio curves, it is equally well applicable to true stress vs. stretch ratio curves since the post-necking fiber diameter was shown to be independent of strain rate.

The good fitting of the present data to Equation (2) provides experimental support to theoretical and computational studies reported in the recent years on the nature of the strain hardening process in glassy polymers. Although the strain hardening response of glassy polymers was originally modeled as a rate independent, non-dissipative, rubber elastic process [11], several studies in the last decade have supported the rate-dependent nature of the strain hardening [40-47]. Theoretical studies have suggested that the strain hardening behavior can be considered as the outcome of anisotropic chain conformations due to large deformation which modified the inter-chain packing leading to increased dynamic constraints and prolonged segmental relaxations, thereby making it difficult to overcome the energy barrier for activated hopping mechanisms to occur [41]. Similarly, molecular simulations $[40,45]$ which reported the validity of Equation 2 have suggested that strain hardening arises from increasing dynamical 
constraints when more energy is dissipated to permanently rearrange chain segments between entanglements in the direction of applied stress while maintaining inter-chain connectivity of the polymer chain network. Notably, some experimental data from bulk-scale uniaxial compression experiments on amorphous polymers, such as PS and PMMA, obtained over a relatively narrow range of low stain rates $\left(10^{-4}-10^{-2} \mathrm{~s}^{-1}\right)$ have been used to argue that the strain hardening response of bulk polymer glasses is influenced by both entropic elasticity and viscous dissipation mechanisms, since Equation 2 could not adequately describe the experimental data at hand [42]. In contrast, the experimental results presented in this work for PS nanofibers with $D_{\text {norm }}<10$ consistently follow the scaling of Equation 2 in a broad range of strain rates $\left(10^{-4}-10^{2} \mathrm{~s}^{-1}\right)$ and demonstrate the validity of Equation 2 in the entire loading regime that includes necking and post-neck hardening.

In a previous study by the authors [8], it was shown that the stress vs. stretch ratio

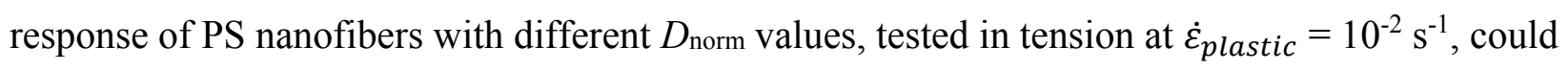
be collapsed onto a single curve, by proper normalization of the fiber stretch ratio. The scaling of the viscoplastic response of PS nanofibers shown in Figures 7 and 8, could be combined with the scaling analysis in [8] to obtain a unifying description for the role of spatial confinement (through the use of $D_{\text {norm}}$ ) and strain rate on the elasto-plastic response of PS at the submicron scale, by re-plotting the curves in Figures 7(b) and 8(a-c) in normalized stress and strain axes, as shown in Figure 9. It is worth noting that the value $\varepsilon_{\text {norm,h }} \approx 0.57$ of normalized strain at the completion of necking shown in Figure 9 is in good agreement with that reported in previous studies $\left(\varepsilon_{\text {norm,h }} \approx 0.61\right)[8,48]$. The curves in Figure 9 , follow a single master curve that simultaneously captures the intertwined temporal and molecular length scale/specimen size ( $\left.D_{\text {norm}}\right)$ effects on fiber extension until the completion of necking, but diverge in the post-neck 
strain hardening regime: decreasing values of $D_{\text {norm }}$ lead to more pronounced post-neck hardening in the normalized stress-strain space, pointing out to molecular orientation taking place during necking.

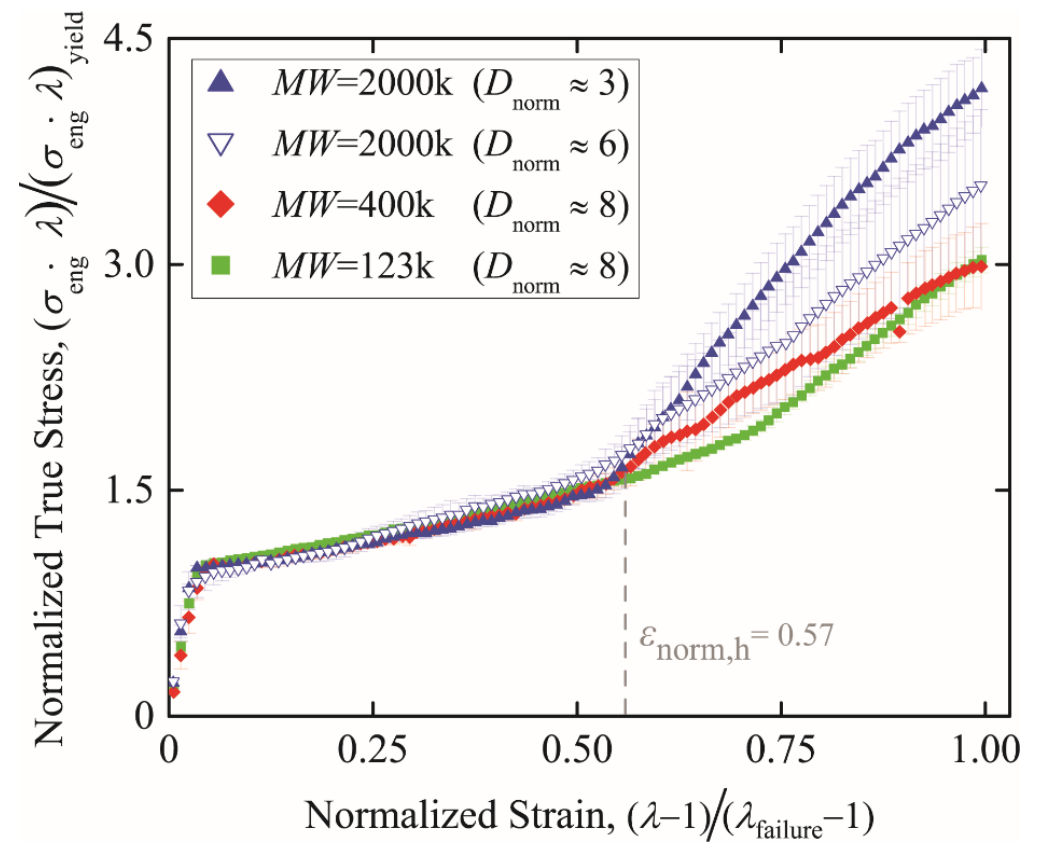

Figure 9. Normalized true stress vs. normalized strain curves using the curves in Figures 3(a) $[M W=$ 2,000,000 g/mol, $\left.D_{\text {norm }}=3\right], 3$ (b) $\left[M W=2,000,000 \mathrm{~g} / \mathrm{mol}, D_{\text {norm }}=6\right], 3(\mathrm{c})\left[M W=400,000 \mathrm{~g} / \mathrm{mol}, D_{\text {norm }}\right.$ $=8]$, and 3(d) $\left[M W=123,000 \mathrm{~g} / \mathrm{mol}, D_{\text {norm }}=8\right]$. The error bars represent the standard error of the mean.

\section{Conclusions}

The strain rate dependent large deformation behavior of individual PS nanofibers with molecular weights in the range $123,000-2,000,0000 \mathrm{~g} / \mathrm{mol}$ and diameters of $150-900 \mathrm{~nm}$ was studied under uniaxial tension, across a wide range of engineering strain rates $\left(10^{-4}-10^{2} \mathrm{~s}^{-1}\right)$. PS 
nanofibers with $D_{\text {norm }}<10$ consistently demonstrated stable necking and subsequent post-neck hardening with little change in $\lambda_{\mathrm{h}}$ at different strain rates, but with significant increase in the yield/flow stress, thus resulting in simultaneous strengthening and toughening. For nanofibers with $D_{\text {norm }}<10$, the complete engineering stress vs. stretch ratio response was shown to scale well with yield stress in the entire range of strain rates applied in this study, thus obeying a multiplicative decomposition of stress into a shape and a rate component. A master curve composed of normalized stress and strain axes was shown to capture well the temporal and specimen size/molecular length scale effects until the completion of necking, while the post-neck

hardening response was shown to be $D_{\text {norm }}$ dependent, as a result of molecular orientation taking place during necking. On the other hand, nanofibers with $D_{\text {norm }}>10$ demonstrated a monotonic increase in yield strength with strain rate, but did not sustain stable necking, often failing prior to the complete propagation of a neck in the entire gage length. In fact, nanofibers with $D_{\text {norm }} \approx 18$, demonstrated a ductile-to-brittle transition upon increasing the elastic strain rate from $1 \mathrm{~s}^{-1}$ to 30 $\mathrm{s}^{-1}$.

\section{Acknowledgments}

This material is based upon work supported by the National Science Foundation under the PECASE Grant No. 0748120. 


\section{References}

[1] Haward, R.N., Young, R.J., Eds.; The Physics of Glassy Polymers, $2^{\text {nd }}$ ed.; Chapman and Hall: London, 1997.

[2] Bucknall, C.B. Toughened Plastics; Applied Science Publishers: London, 1977.

[3] Riew, C.R., Kinloch, A.J., Eds.; Toughened Plastics I. Advances in Chemistry Series 233; American Chemical Society: Washington D.C., 1993.

[4] C.R. Riew, C.R., Kinloch, A.J., Eds.; Toughened Plastics II. Advances in Chemistry Series 252; American Chemical Society: Washington D.C., 1996.

[5] Brown, H.R.; Argon, A.S.; Cohen, R.E.; Gebizlioglu, O.S.; Kramer, E.J. Macromolecules 1989, 22, 1002-1004.

[6] Meijer, H.E.H.; Govaert, L.E. Prog. Polym. Sci. 2005, 30, 915 - 938.

[7] Zartman, G.D.; Cheng, S.; Li, X.; Lin, F.; Becker, M.L.; Wang, S.-Q. Macromolecules 2012, 42, $6719-6732$.

[8] Kolluru, P.V.; Chasiotis, I. Polymer 2015, 56, 507-515.

[9] Boyer, R.F. Polym. Eng. Sci. 1968, 8, 161-185.

[10] Argon, A.S. The Physics of Deformation and Fracture of Polymers, Cambridge University Press: New York, 2013.

[11] Mulliken, A.D.; Boyce, M.C. Int. J. Solids Struct. 2006, 43, 1331-1356.

[12] van Breemen, L.C.A.; Engels, T.A.P.; Klompen, E.T.J.; Senden, D.J.A.; Govaert, L.E. J. Polym. Sci., Part B: Poly. Phys. 2012, 50, 1757-1771.

[13] Haward, R.N.; Thackray, G. Proc. R. Soc. Lond., Ser. A: Math Phys. Sci. 1968, 302, 453-472.

[14] Imai, Y; Brown, N. J. Polym. Sci., Part B: Polym. Phys. 1976, 14, 723-739.

[15] Boyce, M.C.; Arruda, E.M. J. Polym. Eng. Sci. 1990, 30, 1288-1298.

[16] Arruda, E.M.; Boyce, M.C.; Jayachandran, R. Mech. Mater. 1995, 19, 193-212.

[17] Sarva, S.S.; Boyce, M.C. J. of Mech. Mater. Struct. 2007, 2, 1853-1880.

[18] O’Connell, P.A.; Hutcheson, S.A.; McKenna, G.B. J. Polym. Sci., Part B: Polym. Phys. 2008, 18, 1952-1965.

[19] Chan, E.P.; Page, K.A.; Im, S.H.; Patton, D.L.; Huang, R.; Stafford, C.M. Soft Matter 2009, 5, $4638-4641$. 
[20] Rowland, H.D.; King, W.P.; Cross, G.L.; Pethica J.B. ACS Nano 2008, 2, 419-428.

[21] Fukao, K.; Miyamoto, Y. Phys. Rev. E. 2000, 61, 1743-1754.

[22] Fukao, K.; Miyamoto, Y. Phys. Rev. E 2001, 64, 011803.

[23] Fukao, K; Uno, S.; Miyamoto, Y.; Hoshino, A.; Miyaji, H. Phys. Rev. E 2001, 64, 051807.

[24] Wubbenhorst, M.; Murray, C.A.; Dutcher, J.R. Eur. Phys. J. E: Soft Matter 2003, 12, S109-112.

[25] Alcoutlabi, M.; McKenna, G.B. J. Phys.: Condens. Matter 2005, 17, R461-R524.

[26] Kim, S.; Hewlett, S.A.; Roth, C.B.; Torkelson, J.M. Eur. Phys. J. E 2009, 30, 83-92.

[27] Kim, S.; Torkelson, J.M. Macromolecules 2011, 44, 4546-4553.

[28] Paeng, K.; Swallen, S.F.; Ediger, M.D. J. Am. Chem. Soc. 2011, 133, 8444-8447.

[29] O’Connell, P.A.; McKenna, G.B. Science 2005, 307, 1760-1763.

[30] Naraghi, M.; Kolluru, P.V.; Chasiotis, I. J. Mech. Phys. Solids 2014, 62, 257-275.

[31] Kolluru P.V.; Lipner J.; Wenying L.; Xia Y.; Thomopoulos S.; Genin G.M.; Chasiotis I. Acta Biomat, 2013, 9, 9442-9450.

[32] Naraghi, M.; Chasiotis, I.; Kahn, H.; Wen, Y.; Dzenis, Y. Rev. Sci. Instrum. 2007, 78, 085108.

[33] Naraghi, M.; Chasiotis, I.; Dzenis, Y. Wen, Y.; Kahn, H. App Phys Lett 2007, 91, 151901.

[34] Naraghi, M.; Chasiotis, I. J. Microelectromech. Syst. 2009, 18, 1032-1046.

[35] Ji, Y.; Li, C.; Wang, G.; Koo, J.; Ge, S.; Li, B.; Jiang, J.; Herzberg, B.; Klein, T.; Chen, S.; Sokolov, J.C.; Rafailovich, M.H. Europhys. Lett. 2008, 84, 56002.

[36] Sussman, D.M.; Tung, W.-.S.; Winey, K.I.; Schweizer, K.S.; Riggleman, R.A. Macromolecules 2014, 47, 6462-6472.

[37] Tung, W.-.S.; Composto, R.J.; Riggleman, R.A.; Winey, K.I. Macromolecules 2015, 48, 23242332.

[38] Meijer, H.E.H.; Govaert, L.E.; Smit, R.J.M. In Toughening of Plastics: Advances in Modeling and Experiments; Pearson, R.A.; Sue H.-J.; Yee, A.F., Eds.; ACS Symposium Series 759; American Chemical Society: Washington D.C., 2000.

[39] Eyring, H. J. Chem. Phys. 1936, 4, 283-291.

[40] Hoy, R.S.; Robbins, M.O. J. Polym. Sci., Part B: Polym. Phys. 2006, 44, 3487-3500.

[41] Chen, K.; Schweizer, K.S. Phys. Rev. Lett. 2009, 102, 038301. 
[42] Govaert, L.E.; Engels, T.A.P.; Wendlant, M.; Tervoort, T.A.; Suter, U.W. J. Polym. Sci., Part B: Polym. Phys. 2008, 46, 2475-2481.

[43] Robbins, M.O.; Hoy, R.S. J. Polym. Sci., Part B: Polym. Phys. 2009, 47, 1406-1411.

[44] Coates, P. D.; Ward I. M. J Mat. Sci. 1980, 15, 2897-2914.

[45] Hoy, R.S.; Robbins, M.O. Phys. Rev. Lett. 2007, 99, 117801.

[46] Dupaix, R.B.; Boyce, M.C. Polymer 2005, 46, 4827-4838.

[47] Wendlant, M.; Tervoort, T.A.; Suter, U.W. Polymer 2005, 46, 11786-11797.

[48] Rottler, J.; Robbins, M.O. Phys. Rev. E 2003, 68, 011801. 